\title{
A Monte Carlo intercomparison of peak-to-valley dose ratios and output factors for microbeam radiation therapy
}

\author{
Dimitri Reynard ${ }^{1,2}$, Richard P. Hugtenburg ${ }^{2,3}$ \\ 1- Université Grenoble Alpes, EA 7442 RSRM - ID17/ESRF-CLUNI \\ 2- Swansea University, Singleton Park Campus, Sketty, Swansea, SA2 8PP, United \\ Kingdom \\ 3- Singleton Hospital, Swansea Bay University Health Board, Swansea SA2 8QA
}

\begin{abstract}
A comparison between EGSnrc, Penelope and Geant4 has been made for dosimetry applied to Microbeam Radiation Therapy (MRT). A simple geometry is defined to limit the number of influential parameters and to focus primarily on the dose associated with scattered photons. Use was made of a precalculated photon spectrum for the ESRF ID17 Medical beamline ranging from 40-300 keV with a mean energy of $107 \mathrm{keV}$. In MRT, Compton scattering is the main photon interaction in soft tissue, with the photoelectric effect contributing more substantially in bone. The study investigates differences in the way Compton scattering is handled by the different codes which lead to differences of up to $4 \%$ for the simulation of relevant dosimetric quantities in MRT, despite the fact that the cross-section data comes from the same source. There is no significant pattern in the way the codes behave and depending on the dosimetric quantity involved, the agreement between the codes varies. The agreement for each dosimetric quantities is enhanced at
\end{abstract}


large depths where beam-hardening increases the mean energy of the beam and lowers the influence of Doppler broadening and electron binding effects, allowing the codes to use less corrections to the Klein-Nishina model which the three codes implement in the same way.

Keywords: Microbeam radiation therapy, Monte Carlo, Compton

scattering

\section{Introduction}

Microbeam Radiation Therapy (MRT), an emergent treatment modality that utilises an array of narrow microbeams to treat malignant brain tumours, is currently in the pre-clinical phase of development at the ID17 biomedical beamline of the European Synchrotron Radiation Facility (ESRF). The microbeams are $50 \mu \mathrm{m}$ wide, spaced with $400 \mu \mathrm{m}$ and the energy beam ranges from 40 to $300 \mathrm{keV}$ [1]. The challenge with such spatially fractionated irradiation fields lies in the development of a reliable experimental dosimetry, but there are also challenges in dose calculations [2][3][4].

The peak dose deposited on the path of the microbeams is mainly due to primary interactions and subsequent transport of electrons from the peak, while the valley dose (the dose deposited between the peaks) is the consequence of Compton scattering for the most part. Therefore accurate modelling of Compton scattering and electron transport on the micron scale is required. Thus far there have been inconsistencies in the result from the Monte Carlo (MC) codes used to predict two dosimetric quantities used in 
microbeam dosimetry and planning; the output factor (OF), which is the dose in the peak versus the dose for an equivalent broad field suitable for dose measurements with an ionisation chamber and the peak-to-valley-ratio (PVDR), the ratio of dose in peak to the diffuse dose distribution between peaks. The main motivation for this work are discrepancies between output factor simulations for MRT in the literature comparing Penelope and Geant4 where the level of discrepancy prevents meaningful comparison with experiment[2].

This study involves three Monte Carlo codes; EGSnrc [5, 6], Penelope [7][8][9] and GATE [10]which implements its radiation transport with Geant4 in a convenient voxel geometry.

The Compton scattering models used for EGSnrc and Penelope are based on Ribberfor's scattering model, where a double-differential Compton scattering cross-section (DDCS) is used in the relativistic impulse approximation (RIA) [9]. The method was developed to account for the scattering of a bound electron considering its binding energy and pre-collision momentum of the target electron [11] as opposed to use of the simple Klein Nishina formula for free electron scattering. This binding effect becomes noticeable in term of Doppler broadening of the scattered photon's energy distribution for energies lower than $5 \mathrm{MeV}$ and hence has to be accurate in MRT. For EGSnrc, the user has the choice between using the free electron approximation (Klein-Nishina) or the relative impulse approximation to allow binding effect and Doppler Broadening consideration. EGSnrc relies on published cross-section data from Storm and Israel (1970), EPDL (Cullen et al 1997) 
[12] or Berger and Hubbell, 1987 [13] depending on the user's choice. In Penelope Compton scattering is simulated using the algorithm from Brusa [7] using the impulse approximation which accounts for the binding effect and Doppler broadening below $5 \mathrm{MeV}$. The cross-section data used is from EPDL (Cullen et al 1997). For GATE/Geant4 the user can define which physics processes are used in a calculation through the "physics list". In this study the Geant4 physics list G4EmStandardPhysics-option 4 is adopted. In terms of Compton scattering modelling, in the energy range relevant to this study the G4LowEPComptonModel [14] is used. The G4LowEPComptonModel utilises the Monash University Compton scattering algorithm as an alternative to Ribberfor-based algorithms. The cross-section data are also from EPDL (Cullen et al 1997).

All three codes use a class II condensed history technique for the simulation of charged particle transport, although the implementations differ somewhat. In particular, EGSnrc uses the parameter reduced electron-step transport algorithm (PRESTA) that means that in principle it is not necessary to set a minimum step-length. Electron transport in GEANT4 is implemented using a Goudsmit-Saunderson model [15]. Step-lengths are predefined in Geant4, and should therefore be chosen to be significantly smaller than any features in the simulation. Penelope uses a mixed algorithm in which hard events, defined as those involving angular deflections or energy loses above a certain user-defined threshold, are simulated in an analogue way; the events are simulated interaction-by-interaction. The combined effect of all the soft interactions occurring between two consecutive hard collisions is accounted for by means of a single artificial event. The maximum step-length is con- 
trolled by setting DSMAX following the guidance that the step length should be one tenth of the smallest dimension of a region. The multiscattering technique used by Penelope has been tested over a wide range of energies [16].

Microbeam dosimetry has been studied in the past using MC simulations [17] [18][19] [20]. A variety of codes have been investigated including Geant4 [21] [22] [23], EGS4 [24] [17], EGS5 [25] and Penelope [3][18][19]. De Felici [26] compared EGS4, EGSnrc, Penelope, Geant4 and MCNPX using the same irradiation conditions and geometries involving $25 \mu \mathrm{m}$ wide cylindrical and rectangular microbeams. A maximum difference in the dose profile in the range 10 to $100 \mu \mathrm{m}$ of $20 \%$ was found for MCNPX and $19 \%$ for EGS4 and Geant4. The authors limited their study to a maximum range from 0 to 1 $\mathrm{mm}$ away from the microbeam axis with $1 \mu \mathrm{m}$ wide bins, which therefore did not provide information for radiation scattering from the edge of the field. Scattered radiation from the edge of the field, which can be more than 10 $\mathrm{mm}$ in clinically relevant beams, contributes significantly to output factors and peak-to-valley dose ratios, both quantities of interest in MRT to assess the therapeutic outcome and dose delivery during the treatment.

The aim of this study is to compare calculations of the dosimetric parameters, OF and PVDR, using simulations in a relatively simple and reproducible geometry. The work examines the agreement between three major Monte Carlo codes, where with a systematic choice of geometry and source description other sources of divergence have been eliminated, enabling the focus to be on the Monte Carlo implementation, including choice of transport models and cross-sections. 


\section{Material and Methods}

The comparison of the MC codes is performed using a rather simple geometry. The method of collimation is ignored and assumes parallel rectan-

gular microbeams and thereby facilitates use of an adjoint method for rapid calculations. The resulting calculation is highly efficient, yet preserves the important aspects of the differences of implementation, enabling comparisons between the codes to be made at high levels of statistical precision. Water and bone (ICRU-44) [27] phantoms are constructed that are $6 \mathrm{~cm} \times 6 \mathrm{~cm}$ $\times 20 \mathrm{~cm}$, with the geometry divided into 1200 slices of $50 \mu \mathrm{m}$ width in the $\mathrm{X}$ direction, 3 slices of $2 \mathrm{~cm}$ wide in $\mathrm{Y}$ and 125 slices of $0.16 \mathrm{~cm}$ width the in $\mathrm{Z}$ direction. The dimensions of the phantom were chosen to reduce computation associated with particles that are unlikely to return to the sensitive volume. It is found that the choice of phantom size introduces a $0.5 \%$ error in the first $5 \mathrm{~cm}$ of depth rising to $1 \%$ approaching $10 \mathrm{~cm}$ depth compared to an effectively, semi-infinite water phantom.

The photon source is an infinitesimal pencil beam which enters the geometry travelling in the positive-Z direction, centred on pixel 601 of the $\mathrm{X}$ axis. Accordingly the integrated energy deposition of the pencil beam in a 50 $\mu \mathrm{m}$ by $2 \mathrm{~cm}$ region is equivalent to the deposition of a $50 \mu \mathrm{m}$ by $2 \mathrm{~cm}$ beam at the position of the pencil beam, by the reciprocal relations implicit in the geometry. The photon spectrum is taken from Martinez et al., 2012,[3], who modelled the major aspects of the ESRF ID17 beam-line, including the filtration and collimation used typically in a clinical setting, and $10^{8}$ photons are used in each simulation. The dosimetric factors are reported at $2 \mathrm{~cm}$ depth, which is precisely the middle of the $13^{\text {th }}$ slice in the $\mathrm{Z}$ direction. 
The single microbeam profile obtained from the simulation is then used to calculate OF and PVDR dosimetric parameters for an array of microbeams. The calculation of OF from a single microbeam profile can be achieved because a full field is the sum of any number of $50 \mu \mathrm{m}$ wide microbeams required to obtain the desired field size. By adding up the contribution of each voxel from the profile to the central pixel (601) one obtains the dose for a $2 \times$ $2 \mathrm{~cm}^{2}$ field. For the calculation of the PVDR, it is mandatory to account for the centre-to-centre distance $(400 \mu \mathrm{m})$ by adding the contribution from each microbeam contained in every 8th pixel of the single microbeam profile at peak and valley positions. Monte Carlo calculations were also performed with electron transport disabled by selecting a cut-off value for the electrons equal to the maximum energy in the spectrum. This way the electron energy is deposited entirely at the location of the interaction.

Electron transport with EGSnrc used the PRESTA-II algorithm with a maximum fractional energy loss of $25 \%$ and exact boundary crossing. The electron and photon transport cut-offs were chosen to be $10 \mathrm{keV}$. The calculation time for $10^{8}$ initial photons was 53 minutes on a single Intel(R) Core(TM) i7-6700 CPU running at 3.40GHz. The Penelope calculations used DSMAX $=10^{-5} \mathrm{~cm}$, the electron and photon transport cutoff were also $10 \mathrm{keV}$, with $\mathrm{C} 1=0.05, \mathrm{C} 2=0.05, \mathrm{WCC}=500 \mathrm{eV}$ and $\mathrm{WCR}=10 \mathrm{eV}$. The calculation times for Penelope were approximately 300 minutes on the same architecture. The Geant4 calculations also used length-based cut-offs of 1 micron for all particles. The calculation times were approximately 245 minutes. 


\section{Results}

Output factors (OF) and peak-to-valley dose ratios (PVDR) are subject to statistical uncertainties of $0.26 \%$ and $1.2 \%$, respectively, at 2 standard deviations, where the uncertainty in the ratios is calculated using the square root of the quadratic sum of the relative uncertainties. Table 1 provides the results of the comparison of $\mathrm{OF}$ and PVDR values for the three codes.

The comparison of OF from EGSnrc, Geant4 and Penelope with electron transport switched on presents differences of up to 2.0\%. Geant4 agrees with EGSnrc within the uncertainty bars but Penelope generates a lower OF. PVDR are in agreement to the level of uncertainty for all codes, where the uncertainty is somewhat higher than the OF calculations. In bone, both Geant4 and Penelope present a higher OF, with differences of up to $2.6 \%$ from EGSnrc and even higher for PVDR, with differences of up to $3.9 \%$.

The maximum difference for the primary dose along the central pixel for water and bone and irrespective of whether electron transport is on or off is less than $0.05 \%$ suggesting that the difference in OF and PVDR values between the codes comes from either photon scattering of electron transport or a combination of both, but not the primary interactions.

Switching off electrons enables the examination of effects due to Compton interaction processes and photoelectric absorption. Both Geant4 and Penelope give higher OF and PVDR values than EGSnrc in water when electron transport is switched off, suggesting that differences in electron transport between the codes is masking differences in the handling of photons. The agreement in $\mathrm{OF}$ values between the codes is however within $1 \%$ and the 
differences in the PVDR calculation are within 1.9\% for Geant4, which are larger than the uncertainty bars. For bone the agreement is poorer with a maximum relative difference to EGSnrc of $1 \%$ for the OF calculation and $3.3 \%$ for the PVDR.

Figure 1 displays the OF for water and bone for each code and the percentage difference in OF and PVDR with respect to EGSnrc with depth. Error-bars are not displayed for better readability. The statistical uncertainty is evident in the dispersion to what would otherwise be smooth curves. Regarding the OF in water, Geant4 and EGSnrc have a close behaviour with higher values than Penelope. The agreement between the codes improves with the removal of electron transport for both water and bone, to better than $1 \%$, worsening only slightly with depth. Similar trends are observed for the PVDR which is, however, more sensitive to the statistical uncertainty in the Monte Carlo calculation because only one point (the peak dose) appears in the numerator of the dose ratio. In bone, the OF trend is inverted giving Penelope and Geant4 closer agreement, leaving EGSnrc with a lower value. The agreement worsens with depth but appears to reach a maximum discrepancy of around $2.5 \%$.

Although all of the codes use the same cross-sectional data for Compton scattering, a potential explanation of the differences in is addressed in "A Survey of Photon Cross Section Data for use in EPICS2017" by Dermott.E Cullen published in February 2018 [28]. In Penelope, a normalisation screening correction is applied at low energy cross-sections. It normalises the bound 


\begin{tabular}{|c|c|c|c|c|c|c|}
\hline & EGSnrc & Geant4 & cf. EGSnrc & Penelope & cf. EGSnrc & $\begin{array}{l}\text { Irradiation } \\
\text { conditions }\end{array}$ \\
\hline $\mathrm{OF}$ & 0.7404 & 0.7376 & $-0.38 \% \pm 1 \%$ & 0.7254 & $-2.02 \% \pm 1 \%$ & $2 \times 2 \mathrm{~cm}^{2} 2 \mathrm{~cm}$ \\
\hline PVDR & 28.9 & 29.23 & $+1.17 \% \pm 2.1 \%$ & 28.24 & $-1.63 \% \pm 2.1 \%$ & depth in water \\
\hline $\mathrm{OF}$ & 0.7914 & 0.7960 & $+0.58 \% \pm 1 \%$ & 0.7931 & $+0.21 \% \pm 1 \%$ & $2 \mathrm{x} 2 \mathrm{~cm}^{2} 2 \mathrm{~cm}$ \\
\hline PVDR & 31.44 & 32.03 & $++1.89 \% \pm 2.1 \%$ & 31.5 & $+0.39 \% \pm 2.1 \%$ & $\begin{array}{c}\text { depth in water } \\
\text { no electron }\end{array}$ \\
\hline OF & 0.5470 & 0.5614 & $+2.63 \% \pm 1 \%$ & 0.5585 & $+2.10 \% \pm 1 \%$ & $2 \times 2 \mathrm{~cm}^{2} 2 \mathrm{~cm}$ \\
\hline PVDR & 12.19 & 12.66 & $+3.87 \% \pm 2.1 \%$ & 12.63 & $+3.67 \% \pm 2.1 \%$ & depth in bone \\
\hline OF & 0.6124 & 0.6164 & $+0.65 \% \pm 1 \%$ & 0.6275 & $+2.47 \% \pm 1 \%$ & $2 \mathrm{x} 2 \mathrm{~cm}^{2} 2 \mathrm{~cm}$ \\
\hline PVDR & 13.51 & 13.85 & $+2.51 \% \pm 2.1 \%$ & 13.95 & $+3.26 \% \pm 2.1 \%$ & $\begin{array}{c}\text { depth in bone } \\
\text { no electron }\end{array}$ \\
\hline
\end{tabular}

Table 1: Output factors (OF) and peak-to-valley ratios (PVDR) at $2 \mathrm{~cm}$ depth in water and bone for EGSnrc,Geant4 and Penelope, with percent differences for the latter two in comparison with EGSnrc, including calculations where electron transport has been switched off. 


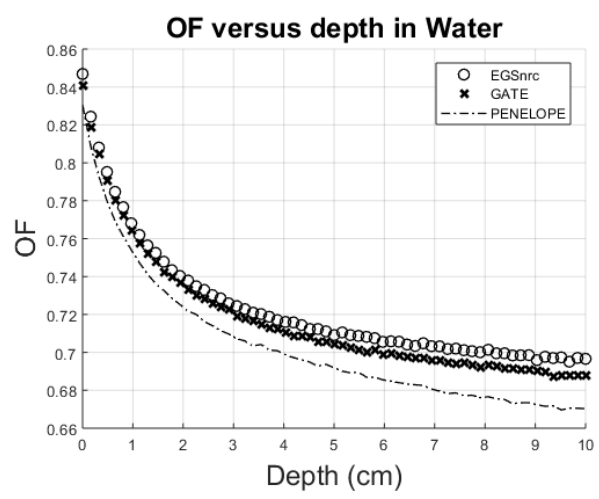

OF versus depth in Water no electron transport

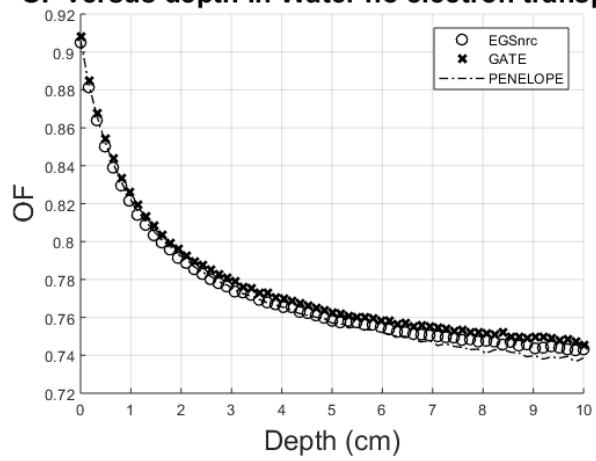

Water

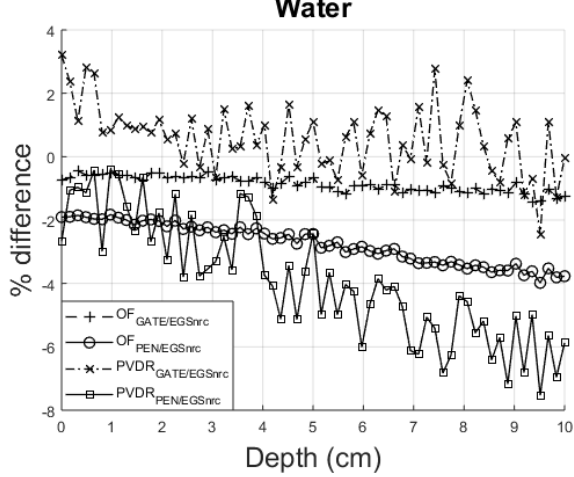

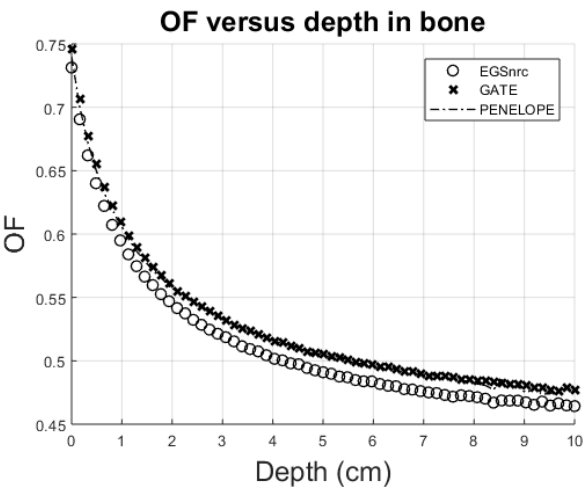

OF versus depth in bone no electron transport

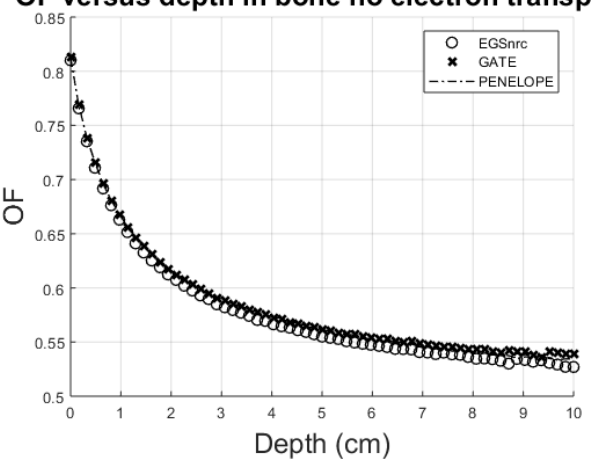

Bone

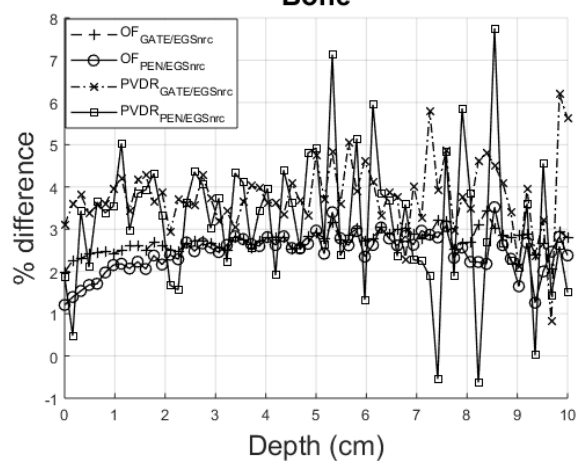

Figure 1: OF factors as a function of depth for EGSnrc, Geant4 and Penelope Monte Carlo codes, in water and bone media, and with and without electron transport. Percentage comparisons of OF and the PVDR factors with EGSnrc are given in the lower 2 panels 
states of the atom compared to a bare nucleus with an energy independent factor accounting for the electron density. This has the consequence of lowering the photoelectric cross section and increasing the Compton cross-section which explains the increase in scattered radiation in the case of Penelope. The difference to the photoelectric effect cross-section for light elements is of the order of $1-2 \%$. The effect in water will not be pronounced, given that photoelectric effect is less than $2 \%$ of the total cross-section. In bone, however, the contribution from the photoelectric effect to the cross-section is approximately $25 \%$. It might therefore be possible to observe the differences in beam penumbra, due to different proportions of photoelectrons predicted by the models. Cullin states that considering the lack of experimental data, the use of the normalisation is allowed as theory allows it, but its relevance is not proved yet.

With regard to the influence of electron transport, the simulations highlight a difference in close vicinity to the microbeams, with a $30 \%$ increase in the dose deposition in the first pixels (distance 25-75 $\mu \mathrm{m}$ ) around the central pixel for Geant4 and Penelope compared to EGSnrc, however in bone this difference disappears. The results are reminiscent of differences of up to $20 \%$ reported by De Felici [26] comparing dose profiles for distances between 10-100 microns for Penelope and Geant4. As such, the Geant4 physics libraries implemented in the current study use a similar electron transport routine to Penelope, while EGSnrc utilises the parameter reduced electronstep transport algorithm (PRESTA). The principal advantage of PRESTA is an improved speed of simulation, and for Penelope and Geant4, small maximum electron step-sizes are needed through-out the geometry in order that 
boundary-crossing effects are insignificant.

\section{Conclusion}

Three widely used Monte Carlo codes have been benchmarked for dosimetry at the micron scale in order to isolate causes of discrepancy between experimental and simulated results. Our investigation led to the assessment that the codes agree with each other to within $2 \%$ for OF and PVDR calculations in water, which would be considered to be a clinically tolerable level of uncertainty. A small discrepancy between Penelope and EGSnrc exists for the OF calculation in water that may be attributed to differences in the handling of Compton scattering. Discrepancies in PVDR and OF increase in bone, supporting the conclusion that differences in the implementation of the photoelectric may be important. In general larger discrepancies were observed with electron transport switched on and effort should be made in future studies to address the differences in electron transport occuring at micron dimensions.

The codes are also in poorer agreement in bones, an aspect that should be investigated further as it may become an issue with the use of more

realistic phantoms containing cortical and cancellous bone, and in clinical implementations.

\section{Acknowledgement}

DR would like to acknowledge the part-funding of his $\mathrm{PhD}$ programme of study by the Swansea Bay University Health Board. 


\section{References}

[1] E. Bräuer-Krisch, J.-F. Adam, E. Alagoz, S. Bartzsch, J. Crosbie, C. DeWagter, A. Dipuglia, M. Donzelli, S. Doran, P. Fournier, et al., Medical physics aspects of the synchrotron radiation therapies: Microbeam radiation therapy (mrt) and synchrotron stereotactic radiotherapy (ssrt), Physica Medica 31 (2015) 568-583.

[2] Y. Prezado, I. Martínez-Rovira, M. Sánchez, Scatter factors assessment in microbeam radiation therapy, Medical physics 39 (2012) 1234-1238.

[3] I. Martínez-Rovira, J. Sempau, Y. Prezado, Development and commissioning of a monte carlo photon beam model for the forthcoming clinical trials in microbeam radiation therapy, Medical physics 39 (2012) 119131.

[4] P. Fournier, J. C. Crosbie, I. Cornelius, P. Berkvens, M. Donzelli, A. Clavel, A. B. Rosenfeld, M. Petasecca, M. L. Lerch, E. Bräuer-Krisch, Absorbed dose-to-water protocol applied to synchrotron-generated xrays at very high dose rates, Physics in Medicine \& Biology 61 (2016) N349.

[5] Y. Namito, S. Ban, H. Hirayama, Implementation of the Doppler broadening of a Compton-scattered photon into the egs4 code, Nuclear Instruments and Methods in Physics Research Section A: Accelerators, Spectrometers, Detectors and Associated Equipment 349 (1994) 489494 . 
[6] D. W. O. Rogers, I. Kawrakow, J. P. Seuntjens, B. R. B. Walters, E. Mainegra-Hing, NRC user codes for EGSnrc. NRCC Report PIRS702 (Rev. B), NRCC, 2003.

[7] D. Brusa, G. Stutz, J. Riveros, J. Fernández-Varea, F. Salvat, Fast sampling algorithm for the simulation of photon Compton scattering, Nuclear Instruments and Methods in Physics Research Section A: Accelerators, Spectrometers, Detectors and Associated Equipment 379 (1996) $167-175$.

[8] J. Fernández-Varea, D. Liljequist, S. Csillag, R. Räty, F. Salvat, Monte Carlo simulation of $0.1-100 \mathrm{keV}$ electron and positron transport in solids using optical data and partial wave methods, Nuclear Instruments and Methods in Physics Research Section B: Beam Interactions with Materials and Atoms 108 (1996) 35-50.

[9] F. Salvat, J. M. Fernández-Varea, J. Sempau, Penelope-2008: A code system for monte carlo simulation of electron and photon transport, in: the Workshop Proceedings, June.

[10] S. Jan, D. Benoit, E. Becheva, T. Carlier, F. Cassol, P. Descourt, T. Frisson, L. Grevillot, L. Guigues, L. Maigne, C. Morel, GATE v6: a major enhancement of the GATE simulation platform enabling modelling of CT and radiotherapy, Physics in Medicine \& Biology 56 (2011) 881901.

[11] R. Ribberfors, X-ray incoherent scattering total cross sections and 
energy-absorption cross sections by means of simple calculation routines, Physical Review A 27 (1983) 3061.

[12] D. E. Cullen, J. H. Hubbell, L. Kissel, et al., Epdl97: the evaluated photon data library,'97 version, UCRL-50400 6 (1997) 1-28.

[13] M. J. Berger, J. Hubbell, XCOM: Photon cross sections on a personal computer, Technical Report, National Bureau of Standards, Washington, DC (USA). Center for Radiation Research, 1987.

[14] J. Brown, M. Dimmock, J. Gillam, D. Paganin, A low energy bound atomic electron compton scattering model for Geant4, Nuclear Instruments and Methods in Physics Research Section B: Beam Interactions with Materials and Atoms 338 (2014) 77-88.

[15] O. Kadri, V. Ivanchenko, F. Gharbi, A. Trabelsi, Incorporation of the Goudsmit-Saunderson electron transport theory in the geant4 monte carlo code, Nuclear Instruments and Methods in Physics Research Section B: Beam Interactions with Materials and Atoms 267 (2009) 3624-32.

[16] J. Sempau, P. Andreo, Configuration of the electron transport algorithm of PENELOPE to simulate ion chambers, Physics in Medicine and Biology 51 (2006) 3533-48.

[17] I. Orion, A. Rosenfeld, F. Dilmanian, F. Telang, B. Ren, Y. Namito, Monte carlo simulation of dose distributions from a synchrotronproduced microplanar beam array using the egs4 code system4, Physics in Medicine \& Biology 45 (2000) 2497. 
[18] E. Siegbahn, E. Bräuer-Krisch, J. Stepanek, H. Blattmann, J. Laissue, A. Bravin, Dosimetric studies of microbeam radiation therapy (mrt) with monte carlo simulations, Nuclear Instruments and Methods in Physics Research Section A: Accelerators, Spectrometers, Detectors and Associated Equipment 548 (2005) 54-58.

[19] E. Siegbahn, J. Stepanek, E. Bräuer-Krisch, A. Bravin, Determination of dosimetrical quantities used in microbeam radiation therapy (mrt) with monte carlo simulations, Medical Physics 33 (2006) 3248-3259.

[20] D. N. Slatkin, P. Spanne, F. Dilmanian, M. Sandborg, Microbeam radiation therapy, Medical physics 19 (1992) 1395-1400.

[21] J. Stepanek, H. Blattmann, J. Laissue, N. Lyubimova, M. Di Michiel, D. Slatkin, Physics study of microbeam radiation therapy with psiversion of monte carlo code geant as a new computational tool, Medical physics 27 (2000) 1664-1675.

[22] S. Bartzsch, Microbeam Radiation Therapy-physical and biological aspects of a new cancer therapy and development of a treatment planning system, Ph.D. thesis, Heidelberg, 2014.

[23] I. Cornelius, S. Guatelli, P. Fournier, J. C. Crosbie, M. Sanchez del Rio, E. Bräuer-Krisch, A. Rosenfeld, M. Lerch, Benchmarking and validation of a geant4-shadow monte carlo simulation for dose calculations in microbeam radiation therapy, Journal of synchrotron radiation 21 (2014) $518-528$. 
[24] M. De Felici, R. Felici, C. Ferrero, A. Bravin, A. Tartari, M. Gambaccini, Monte carlo assessment of peak-to-valley dose ratio for mrt, Nuclear Instruments and Methods in Physics Research Section A: Accelerators, Spectrometers, Detectors and Associated Equipment 580 (2007) 489492.

[25] R. P. Hugtenburg, A. Adegunloye, D. A. Bradley, X-ray microbeam radiation therapy calculations, including polarisation effects, with the monte carlo code egs5, Nuclear Instruments and Methods in Physics Research Section A: Accelerators, Spectrometers, Detectors and Associated Equipment 619 (2010) 221-224.

[26] M. De Felici, E. Siegbahn, J. Spiga, A. Hanson, R. Felici, C. Ferrero, A. Tartari, M. Gambaccini, J. Keyriläinen, E. Bräuer-Krisch, et al., Monte carlo code comparison of dose delivery prediction for microbeam radiation therapy, in: Journal of Physics: Conference Series, volume 102, IOP Publishing, p. 012005.

[27] K. Goldstone, Tissue substitutes in radiation dosimetry and measurement, in: Icru report 44. international commission on radiation units and measurements, usa (1989), 1990.

[28] D. E. Cullen, A survey of photon cross section data for use in EPICS2017. IAEA Nuclear Data Services, IAEA-NDS-225 (rev.1), IAEA, Vienna, 2018. 